VoL. 47 (1993) [73-78]

\title{
A DIRECT ELLIPSOID METHOD FOR LINEAR PROGRAMMING
}

\author{
Shiquan Wu and Fang Wu
}

This paper indicates how to apply the ellipsoid method directly to Linear Programming problems and proves that this kind of version of the ellipsoid method is almost as good as Karmarkar's type method in the theoretical sense.

\section{INTRODUCTION}

As several authors have noted, the ellipsoid method is in some sense equivalent to Karmarkar's method [6, 7]. Todd and Ye designed the ellipsoid according to the Karmarkar's potential function and proved that the ratio of the volume reduction of the ellipsoids is equal to the ratio of the potential function reduction. In this note, we apply the ellipsoid method directly to Karmarkar's canonical problem and get a nicer complexity bound than the traditional ellipsoid method. This shows, in another way, the relationship between the ellipsoid method and Karmarkar's method.

\section{KARMARKAR'S PROBLEM AND ITS EQUIVALENCE}

We shall consider the following problem

$$
\begin{gathered}
\min \left\{\bar{c}^{T} \mid \bar{x} \in \bar{R}\right\} \\
\bar{R}=\left\{\bar{x} \mid \bar{A} \bar{x}=0, \bar{e}^{T} \bar{x}=n, \bar{x} \geqslant 0\right\}
\end{gathered}
$$

where

\section{Assumptions.}

(1) $\bar{c}^{T} \bar{x}$ is not a constant on $\bar{R}$.

(2) $\bar{A} \in R^{m \times n}$ and $\operatorname{rank}(\bar{A})=m$.

(3) $\bar{A} \bar{e}=0$ (where $\bar{e}$ denotes an n-dimension vector of 1's).

(4) $\bar{A}$ is an integer matrix.

Received 20th December, 1991

We would like to express our gratitude to Professor Michel Balinski and Professor Manfred Padberg for their kindly help, and to Professof Lázló Lovász for supplying us with the transformation in Section 1.

Copyright Clearance Centre, Inc. Serial-fee code: 0004-9729/93 \$A2.00+0.00. 
Now we suppose that $z_{1}, z_{2}, \ldots, z_{n-m-1}$ are the basic solutions of

$$
\begin{aligned}
\bar{A} \bar{x} & =0 \\
\bar{e}^{T} \bar{x} & =0
\end{aligned}
$$

and $A_{1}, A_{2}, \ldots, A_{n-m-1}$ are their normalised orthogonal vectors. Let $A=$ $\left(A_{1}, A_{2}, \ldots, A_{n-m-1}\right)=\left(a_{1}, a_{2}, \ldots, a_{n}\right)^{T}, c=A^{T_{\bar{c}}}$; then the linear mapping

$$
\bar{x}:=\bar{e}+A x
$$

transforms $(\bar{P})$ into

$$
\min \left\{c^{T} x \mid x \in R\right\}
$$

where $R=\{x \mid A x \geqslant-\bar{e}\}=\left\{x \mid a_{1}^{T} x \geqslant-1, a_{2}^{T} x \geqslant-1, \ldots, a_{n}^{T} x \geqslant-1\right\}$.

It is not difficult to see that to transform $(\bar{P})$ into $(P)$ needs at most $(n+1) \times n \times m$ arithmetic operations. Generally speaking, to transform a standard linear programming into Karmarkar's canonical form needs to combine primal and dual problems (see [2]). So the reduced problem $(\mathrm{P})$ has fewer variables than the problem $(\bar{P})$. The following method is actually designed for solving $(P)$.

\section{Direct Ellitpsoid Method For Problem (P)}

In the following, we use $I$ and $\bar{c}_{i}$ to denote the $(n-m-1) \times(n-m-1)$ identity matrix and the $i$ th component of $\bar{c}$.

Algorithm.

Step 1. Set $x_{1}=0, Q_{1}=n(n-1) I, k=1$;

Step 2. set $d= \begin{cases}-a_{i}, & \text { if there exists an } i \text { such that } a_{i}^{T} x_{k}<-1, \\ c, & \text { otherwise; }\end{cases}$

Step 3. set $x_{k+1}=x_{k}-1 /(n-m)\left(Q_{k} d / \sqrt{d^{T} Q_{k} d}\right)$,

$$
Q_{k+1}=\frac{(n-m-1)^{2}}{(n-m-1)^{2}-1}\left(Q_{k}-\frac{2}{n-m} \frac{\left(Q_{k} d\right)\left(Q_{k} d\right)^{T}}{d^{T} Q_{k} d}\right)
$$

set $k=k+1$ and return to Step 2 .

\section{Main Theorem and Lemmas}

MAIN Theorem. Let $\left\{x_{k}\right\}$ be generated by the algorithm, $l^{*}$ be the optimal 
value of $(P)$; then

$$
\min \left\{c^{T} x_{t}-l^{*} \mid x_{t} \in R, t \leqslant k\right\} \leqslant n(n-1)\left(\max _{i} \bar{c}_{i}-\min _{i} \bar{c}_{i}\right) \exp \left(-\frac{k}{2(n-m)^{2}}\right) .
$$

So as

$$
k \geqslant 2(n-m)^{2} L(\bar{A})+2\left[\ln (n)+\ln \left(\max _{i} \bar{c}_{i}-\min _{i} \bar{c}_{i}\right)\right](n-m)^{2}
$$

we have

$$
\min \left\{c^{T} x_{t}-l^{*} \mid x_{t} \in R, t \leqslant k\right\} \leqslant 2^{-L(\bar{A})} .
$$

In order to prove this theorem, we need the following notions and lemmas. Given an integer $q \neq 0$, we need one cell for the sign and $\left[\log _{2}(|q|+1)\right]$ cells for the $\{0,1\}$ string in order to represent its absolute value in binary. For " 0 " or " 1 " we need only one cell. So to encode an integer $q$ we need

$$
L(q):=1+\left[\log _{2}(|q|+1)\right]
$$

cells. We call $L(q)$ the encoding length of $q$. Hence the encoding length of a rational number $r=s / t$ is $L(r)=L(s)+L(t)$.

\section{LEMMA 1.}

(a) For every rational number $r,|r| \leqslant 2^{L(r)}-1$.

(b) For every rational vector $x \in R^{n},\|x\| \leqslant\|x\|_{1} \leqslant 2^{(L(x)-n)}-1$.

(c) For every rational matrix $D \in R^{n \times n},|\operatorname{det}(D)| \leqslant 2^{\left(L(D)-n^{2}\right)}-1$.

Proof: See [3].

LEMma 2. Without loss of generality we assume that $\bar{c}_{1}=\min _{i} \bar{c}_{i}, \bar{c}_{n}=\max _{i} \bar{c}_{i}$.

(1) $n \bar{c}_{1} \leqslant \bar{c}^{T} \bar{x} \leqslant n \bar{c}_{n}$ for $\bar{x} \in \bar{R}$.

(2) $l_{1}:=(n-1) \bar{c}_{1}-\sum_{i=2}^{n} \bar{c}_{i} \leqslant c^{T} x \leqslant l_{2}:=(n-1) \bar{c}_{n}-\sum_{i=1}^{n-1} \bar{c}_{i}$ for $x \in R$.

(3) $R_{1}=\left\{x \mid\|x\|^{2} \leqslant n /(n-1)\right\} \subset R \subset R_{2}=\left\{x \mid\|x\|^{2} \leqslant n(n-1)\right\}$.

Proof: The proof is very direct, so we omit it here.

Lemma 3. Let $E_{k}=\left\{x \mid\left(x-x_{k}\right)^{T} Q_{k}^{-1}\left(x-x_{k}\right) \leqslant 1\right\}$; then

$$
\frac{\operatorname{vol}\left(E_{k+1}\right)}{\operatorname{vol}\left(E_{k}\right)} \leqslant q_{n-m}=\exp \left(-\frac{1}{2(n-m)}\right) \text {. }
$$

Proof: See [3].

LEMMA 4. (Brünn-Minkowski)

Suppose $C_{1}, C_{2} \subset R^{n}$ are two compact convex subsets; then

$$
\left[\operatorname{vol}\left(\theta C_{1}+(1-\theta) C_{2}\right)\right]^{1 / n} \geqslant \theta\left[\operatorname{vol}\left(C_{1}\right)\right]^{1 / n}+(1-\theta)\left[\operatorname{vol}\left(C_{2}\right)\right]^{1 / n}
$$


Proof: See [4, Theorem 46].

Now let us prove the main theorem. For $k \geqslant 1$, denote

$$
\varepsilon_{k}=\min \left\{c^{T} x_{j}-l^{*} \mid j \leqslant k \text { and } x_{j} \in R\right\} .
$$

Since $x_{1} \in R, \varepsilon_{k}$ is well defined.

For $x_{j} \in R$, denote

$$
\begin{aligned}
H_{j} & =\left\{x \mid x \in R, c^{T} x \leqslant c^{T} x_{j}\right\} \\
\varphi(t) & =\left\{x \mid x \in R, c^{T} x \leqslant t\right\} .
\end{aligned}
$$

It follows that, $l^{*}+\varepsilon_{k} \leqslant c^{T} x_{j}$. So we have

$$
\varphi\left(l^{*}+\varepsilon_{k}\right) \subset R \cap H_{j} \text { for any } j \leqslant k \text { with } x_{j} \in R
$$

Denote

$$
U_{k+1}=R \cap_{1 \leqslant j \leqslant k} H_{j}
$$

By the construction of the ellipsoids we know that, for any $k \geqslant 1$, the following conclusions hold:

$$
\begin{aligned}
& E_{k} \cap\left\{x \mid c^{T} x \leqslant c^{T} x_{k}\right\} \subset E_{k+1}, \text { if } x_{k} \in R ; \\
& E_{k} \cap\left\{x \mid a_{i}^{T} x \geqslant a_{i}^{T} x_{k}\right\} \subset E_{k+1}, \text { if } x_{k} \notin R .
\end{aligned}
$$

We assert that

$$
U_{k+1} \subset E_{k+1} \text { for } k \geqslant 1
$$

In fact, for $k=1$, since $x_{1} \in R$ implies $E_{1} \cap\left\{x \mid c^{T} x \leqslant c^{T} x_{1}\right\} \subset E_{2}$ and $R \subset E_{1}$, it follows that

$$
U_{2}=R \cap H_{1} \subset E_{1} \cap H_{1} \subset E_{2}
$$

Now we assume that for $k=h-1$ the conclusion is true, that is,

$$
U_{h} \subset E_{h}
$$

If $x_{h} \in R$, by (3) and (6) we have

$$
U_{h+1}=U_{h} \cap H_{h} \subset E_{h} \cap H_{h} \subset E_{h+1} .
$$

If $x_{h} \notin R$, then it is clear that $R \subset\left\{x \mid a_{i}^{T} x \geqslant a_{i}^{T} x_{h}\right\}$. By (4) and (6) we get

$$
U_{h+1}=U_{h} \subset E_{h} \cap\left\{x \mid a_{i}^{T} x \geqslant a_{i}^{T} x_{h}\right\} \subset E_{h+1},
$$


and the induction is finished.

By (1), (2) and (5) we get

$$
\varphi\left(l^{*}+\varepsilon_{k}\right) \subset U_{k+1} \subset E_{k+1} .
$$

From Lemma 3

$$
\begin{aligned}
{\left[\operatorname{vol}\left(\varphi\left(l^{*}+\varepsilon_{k}\right)\right)\right]^{1 /(n-m-1)} } & \leqslant\left[\operatorname{vol}\left(E_{k+1}\right)\right]^{1 /(n-m-1)} \\
& \leqslant\left(q_{n-m}\right)^{k /(n-m-1)}\left[\operatorname{vol}\left(E_{1}\right)\right]^{1 /(n-m-1)} .
\end{aligned}
$$

On the other hand, for any $x \in R$, by Lemma 2 we have $l^{*} \leqslant l^{*}+\varepsilon_{k} \leqslant l_{2}$. Hence there exists a $\lambda_{k} \in[0,1]$ such that

$$
l^{*}+\varepsilon_{k}=\lambda_{k} l_{2}+\left(1-\lambda_{k}\right) l^{*}
$$

Furthermore,

$$
\left(1-\lambda_{k}\right) \varphi\left(l^{*}\right)+\lambda_{k} \varphi\left(l_{2}\right) \sqsubseteq \varphi\left(l^{*}+\varepsilon_{k}\right),
$$

and the assumption (1) implies $\operatorname{vol}\left(\varphi\left(l^{*}\right)\right)=0$ and $R=\varphi\left(l_{2}\right)$. From Lemma 4 it follows that

$$
\begin{aligned}
{\left[\operatorname{vol}\left(\varphi\left(l^{*}+\varepsilon_{k}\right)\right)\right]^{1 /(n-m-1)} } & \geqslant\left(1-\lambda_{k}\right)\left[\operatorname{vol}\left(\varphi\left(l^{*}\right)\right)\right]^{1 /(n-m-1)}+\lambda_{k}\left[\operatorname{vol}\left(\varphi\left(l_{2}\right)\right)\right]^{1 /(n-m-1)} \\
& =\lambda_{k}\left[\operatorname{vol}\left(\varphi\left(l_{2}\right)\right)\right]^{1 /(n-m-1)} \\
& =\lambda_{k}[\operatorname{vol}(R)]^{1 /(n-m-1)}
\end{aligned}
$$

Therefore

$$
\begin{aligned}
\varepsilon_{k} & =\lambda_{k}\left(l_{2}-l^{*}\right) \\
& \leqslant\left[\frac{\operatorname{vol}\left(\varphi\left(l^{*}+\varepsilon_{k}\right)\right)}{\operatorname{vol}(R)}\right]^{1 /(n-m-1)}\left(l_{2}-l^{*}\right) \\
& \leqslant\left[\frac{\operatorname{vol}\left(E_{k+1}\right)}{\operatorname{vol}(R)}\right]^{1 /(n-m-1)}\left(l_{2}-l^{*}\right) \\
& \leqslant\left(q_{n-m}\right)^{k /(n-m-1)}\left[\frac{\operatorname{vol}\left(R_{2}\right)}{\operatorname{vol}\left(R_{1}\right)}\right]^{1 /(n-m-1)}\left(l_{2}-l_{1}\right) \\
& \leqslant \exp \left(-\frac{k}{2(n-m)^{2}}\right)(n-1)\left(l_{2}-l_{1}\right) \\
& =\exp \left(-\frac{k}{2(n-m)^{2}}\right) n(n-1)\left(\bar{c}_{n}-\bar{c}_{1}\right),
\end{aligned}
$$

and the proof is completed. 


\section{REFERENCES}

[1] L.G. Khachiyan, 'A polynomial algorithm for linear programming', Dokl. Akad. Nauk USSR 244 (1979), 1093-96. Translated in Soviet Math. Dokl. 20, pp. 191-194.

[2] N. Karmarkar, 'A new polynomial-time algorithm for linear programming', Combinatorica 4 (1984), 373-375.

[3] M. Grötschel, L. Lovász and A. Schrijver, Geometric algorithms and combinatorial optimization (Springer-Verlag, Berlin, Heidelberg, New York, 1988).

[4] H.G. Eggleston, Convexity (Cambridge University Press, Cambridge, 1958).

[5] J.L. Goffin, 'Convergence rates of the ellipsoid method on general convex functions', Math. Oper. Res. 8 (1983), 135-150.

[6] M.J. Todd, Improved bounds and containing ellipsoids in Karmarkar's linear programming algorithm, Technical report 721 (Cornell University, 1986).

[7] Y. Ye, 'Karmarkar's algorithm and the ellipsoid method', Oper. Res. Lett. 4 (1987), 177-182.

[8] H.J. Lüthi, 'On the solution of variational inequalities by the ellipsoid method', Math. Oper. Res. 10 (1985), 515-522.

[0] M.W. Padberg and M.R. Rao, The Russian method for linear inequalities and linear optimization, GBA Working Paper (New York University, New York, 1980).

[10] M.W. Padberg and M.R. Rao, The Russian method for linear inequalities II: Approximate arithmetic, GBA Working Paper (New York University, New York, 1980).

Institute of Applied Mathematics

Academia Sinica

Beijing 100080

Chine 